\title{
Pregnancy with Invasive Carcinoma of the Cervix- A Case Report
}

\author{
Nurun Nahar Khanam¹, Shirin Akter Begum², Humaira Alam² \\ ${ }^{1}$ Associate Professor, ${ }^{2}$ Assistant Professor, Department of Obstetrics and Gynaecology, Bangabandhu Sheikh Mujib Medical University, Dhaka
}

\begin{abstract}
:
Carcinoma of the cervix is a preventable female malignancy. Recently, a 30 years old $5^{\text {th }}$ gravid lady was referred to Bangabandhu Sheikh Mujib Medical University (BSMMU) from Jahurul Islam Medical College \& Hospital, Bajitpur, Kishorganj after diagnosing as a case of 32 weeks pregnancy coupled with invasive carcinoma of the cervix. Histopathology of cervical tissue showed moderately differentiated squamous cell carcinoma. Her cervical growth was confined in the anterior lip of the cervix and clinical stage was 1B. She was severely anemic. Her anemia had been corrected by blood transfusion. Injection dexamethasone was given for fetal lung maturity. Renal function tests and all other investigations for surgical fitness were done. At her $34^{\text {th }}$ weeks of pregnancy caesarean section followed by Wertheim's hysterectomy was performed. Baby was alive, birth weight was $2.3 \mathrm{~kg}$, Apgar score was good. Postoperative period was un-event full. At her $23^{\text {rd }}$ postoperative day she was discharged from Obs and Gynae department and referred to Oncology institute for further management. At the time of discharge her baby was healthy.
\end{abstract}

Key Words: Cervical cancer, pregnancy, Wertheim’s hysterectomy.

[BSMMU J 2009; 2(1): 47-49]

\section{Introduction:}

Cervical Cancer is the second most common female malignancy in the world ${ }^{1}$. Around $80 \%$ of women show up with inoperable disease and approximately 233,000 women die out of the disease in each year ${ }^{1}$. In India cervical cancer is the most common cause of death among women between the ages of 20 to 40 years $^{2}$. Carcinoma of the cervix in pregnancy is a curable disease, if detected and treated in the early stages. When the histologic diagnosis is confirmed and the lesion is appropriately staged, the physician must address the difficulties and controversies associated with the various options for therapy ${ }^{3}$. The two standard treatment modalities for invasive cervical cancer; radical surgery and radiotherapy are also available for women diagnosed with cervical cancer in pregnancy ${ }^{4}$. Patient is evaluated individually and a treatment is outlined taking into consideration the patient's emotional, religious, and ethical beliefs. The well being of both the mother and the fetus must be considered and all options of therapy should be discussed with the party in detail outlining the associated benefits and risks. Treatment recommendations for cervical cancer diagnosed in the $1^{\text {st }}$ or the $3^{\text {rd }}$ trimester may be clearer than those for cancers diagnosed in the $2^{\text {nd }}$ trimester. Currently, fetal salvage in the $1^{\text {st }}$ trimester (weeks: 1 to 12 ) is not feasible in women receiving treatment for invasive cervical cancer ${ }^{5}$. In addition, the maternal risk from delaying the

Address for correspondence: Dr. Nurun Nahar Khanam, Associate Professor, Department of Obstetrics and Gynaecology, BSMMU, Dhaka, Bangladesh, E-mail address: naharbsmmu@gmail.com therapy for advanced-stage disease detected in the $1^{\text {st }}$ trimester until fetal maturity is achieved may be excessive. With no available proven alternative treatment to surgery or radiotherapy, both of which will result in fetal demise. The patient may have to make the difficult decision to interrupt an early pregnancy and proceed with definitive therapy.

If definitive surgical therapy is elected in the first trimester, it should be performed with the fetus in situ; the morbidity associated with this procedure is comparable to that for surgery in non-pregnant patients ${ }^{6}$. If radiotherapy is recommended in the first trimester, as in some patients with bulky cervical tumors or advanced-stage disease, the treatment may be initiated with external radiotherapy with the fetus in situ, or it may follow surgical evacuation of the uterine cavity. Spontaneous abortion usually occurs 35 to 45 days following the initiation of external-beam radiotherapy. If spontaneous abortion does not occur by the completion of external-beam therapy, some patients may be candidates for modified radical hysterectomy as completion of therapy or for uterine evacuation followed by brachy therapy to complete definitive radiotherapy ${ }^{7}$.

The second trimester of pregnancy (weeks 13 to 25) is the period of greatest uncertainty to the physician in recommending definitive therapy ${ }^{8}$. Fetal salvage, if at all possible, is exceedingly rare and carries a high neonatal morbidity rate. On the other hand, delaying therapy to achieve fetal maturity may subject the mother to several weeks or even months of treatment delay with the 
theoretical risk of disease progression and maternal compromise.

If the patient elects to interrupt the pregnancy and undertake definitive therapy, a treatment plan similar to the one employed in the first trimester may be utilized. On the other hand, if pregnancy termination is not an acceptable option or if the patient elects to delay definitive therapy to enhance fetal maturity, a target gestational age for fetal delivery in the third trimester is usually determined, and the patient is monitored closely with pelvic examination and possibly imaging studies (magnetic resonance imaging or ultrasound) to assess possible tumor extension until the target date is reached or fetal lung maturity is documented ${ }^{8}$.

Cervical carcinoma diagnosed in the last trimester of pregnancy (week 26 and after) probably results in less difficulty in treatment planning than when diagnosed in either of the first 2 trimesters, primarily because of advancements in neonatology and the improved fetal salvage rate, particularly after 28 weeks of gestation. It is acceptable to most patients and physicians to delay definitive therapy for a few weeks to achieve a better fetal outcome, even in women with advanced lesions ${ }^{9}$.

Once the target gestational age is reached or fetal lung maturity documented, the fetus is delivered and definitive maternal therapy instituted. Since the majority of cervical cancers in pregnancy are early stage (89\% stage IA-IIA) and since the majority of fetuses will be delivered abdominally (74\% overall and $80 \%$ if diagnosed in the third trimester), ${ }^{2}$ definitive surgical therapy, if indicated, may be coordinated with fetal delivery and completed as a 1-stage operation. Definitive therapy for very early lesions (stage 0-IA1), however, may be delayed until 6 weeks postpartum and tailored to the patient's future fertility plans (conization vs total hysterectomy). If definitive radiotherapy is indicated for lesions detected in the third trimester, external-beam radiotherapy may usually start soon after delivery, followed later by brachy therapy, as indicated. Cancer of the cervix is the most prevalent form of cancer, approximately 500,000 new cases detected each year, many of them fatal ${ }^{1}$. Early marriage, early coitus, early childbearing and repeated child births have been associated with the increasing risk of carcinoma cervix ${ }^{10}$. The somatic consequences of repeated pregnancies may also be exemplified in the clear association between the incidence of cancer of the cervix and high parity. Moreover, the natural history of cervical cancer is such that it seems to follow a progressive course from epithelial dysplasia to carcinoma in situ to invasive carcinoma. Fortunately, it is possible to detect it early during a pre-invasive curable stage by the Pap smear test, and to take measures to prevent it from progressing into a life-threatening illness ${ }^{11}$. In view of this, family planning enables women to control the interval between births and limit family size so that close and repeated pregnancies are avoided, and their health is safeguarded ${ }^{12}$.

\section{Case report:}

A 30 years old lady was admitted to Bangabandhu Sheikh Mujib Medical University (BSMMU) after referring from Jahurul Islam Medical College and Hospital Bajitpur, Kishoregong, with the diagnosis of $5^{\text {th }}$ gravida 32 weeks pregnancy and carcinoma of cervix. She had been married for 15 years and got married just at the time of her menarche. Her menstrual cycle was regular. She was a housewife of low socioeconomic class. Her husband was a day labourer. She was para 4, all of them delivered by untrained birth attendant at home. Among her four children two were alive and two died. She had been suffering from per vaginal blood stained discharge and post coital bleeding six months before her last conception but she did not consult with any doctor. During her pregnancy she noticed irregular pervaginal bleeding, at $7^{\text {th }}$ months of gestation the situation became severe. Initially local village doctor treated her. Later on at her 32 weeks of pregnancy she went to Bajitpur Medical College Hospital. Where she under went per speculum examination and cervical tissue biopsy and histopathology. Histopathology of cervical tissue showed moderately differentiated squamous cell carcinoma. Her cervical growth was confined in the anterior lip of the cervix. Staging was done at the same time, which was stage 1B. On admission at BSMMU her hemoglobin level was $7.8 \mathrm{gm} / \mathrm{dl}$. Anemia was corrected by giving fresh blood transfusion and injection Dexamethasone was given for fetal lung maturity. Renal function tests and all other investigations were done

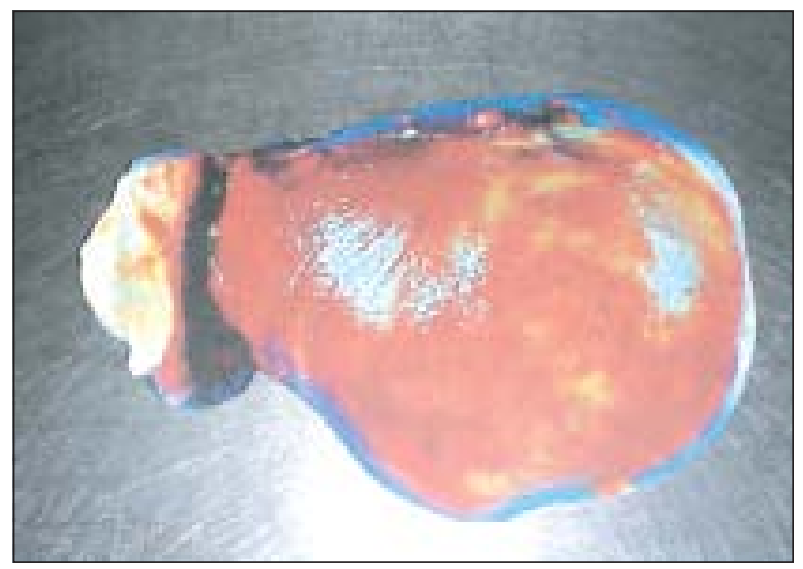

Fig.-1: Specimen of uterus and cervix after operation. 
for surgical fitness. At her 34 weeks of pregnancy caesarean section followed by Wertheim's hysterectomy was done. Baby was alive, birth weight was $2.3 \mathrm{~kg}$, apgar score was good. Maternal condition was good and postoperative period was uneventful. She started exclusive breast-feeding to her baby from first postoperative day. Abdominal wound healed properly. Histopathological reports of the operated specimens were moderately differentiated squamous cell carcinoma involving all layers of the cervix but not uterus or vagina. There were malignant deposit in the left iliac group of lymph nodes; those of the right side were healthy. Consultation with the medical oncologist was done and further management with radiotherapy has been decided. At $23^{\text {rd }}$ postoperative day she was discharged from OBGYN department of the hospital and referred to national oncology institute for further radiotherapy.

\section{Discussion:}

Globally cervical cancer remains an important cause of mortality among young women ${ }^{13}$. Tragically the inequities in health care and competition for scarce funds will mean that the incidence of this disease is unlikely to fall in the near future. There is currently an epidemic of human papilloma virus (HPV) infection and the prospect of a vaccine to this virus becoming available to nonindustrialized countries in next 10 years is small. In industrialized countries, however, the situation is very different with an overall decline in incidence and mortality from cervical cancer. There have been few cases of caesarean section followed by Wertheim's hysterectomy reported in literature. There are several etiological factors, which are responsible for carcinoma cervix such as early marriage, early coitus, early childbearing, and repeated childbirths have been associated with the increasing risk of carcinoma cervix. Invasive cervical cancer during pregnancy is rare but is a dilemma for women and their physicians. The reviews of the literature suggest that pregnancy does not seem to influence the prognosis of cervical cancer ${ }^{10}$. Delayed treatment could be proposed to selected patients diagnosed at the end of the second trimester or at the beginning of the third trimester, with a small tumor $(<2 \mathrm{~cm})$ and negative nodes, after a multidisciplinary approach. Little data exists examining the safety of cesarean section versus vaginal delivery ${ }^{14}$. However, the risks of obstructed labor, hemorrhage and episiotomy site recurrence with vaginal delivery has led to the recommendation of cesarean delivery as the preferred method. Diagnosis of carcinoma of cervix during pregnancy is very difficult and great dilemma as because during pregnancy any kind of pervaginal bleeding considered to be pregnancy related complications. Cervical cancer does not appear to affect the outcome of pregnancy ${ }^{10}$. On investigations her renal function test was normal. All other base line investigations were within normal limit. After caesarean section, Wertheim's hysterectomy was done; patient and her baby were healthy at the time of discharge.

\section{Conclusion:}

Carcinoma cervix is a preventable disease and already has been prevented in most of the developed country. The way of prevention is the secondary form of prevention done by screening out of the disease in its precancerous stage. In our country though some institute have started some screening programs but it has not been spread countrywide. If there were adequate screening program this patient had not been suffered from this disease in her early age. Hopefully this case report will encourage all relevant readers to start cancer-screening program in their institute.

\section{References}

1. Park JE, Park K. Textbook of Preventive and Social Medicine, 19th Ed. Jabalpur, India: M/S Banarasidas Bhanot, 2007. 319-24.

2. World Health Organization: Cytological screening in the control of cervical cancer, Technical Guidelines. WHO; 1988. 52.1.

3. Aras, Radha Y, Rege JD, Pai NP. Screening for carcinoma cervix in a lower socioeconomic class of a peri-urban community. Health and Population- Perspectives and Issues, 1992; 15: 13-15.

4. Saraiya UB. Comparison of cases of cervical cancer, cervical dysplasia and controls with reference to socioeconomic profile and hormonal status. Directory of On-going Research on Cancer Epidemiology, WHO, IARC, Lyon; 1987. 685. 129.

5. Sasmal A. Epidemiology of uterine cervix cancer. Directory of Ongoing Research or Cancer Epidemiology. WHO, IARC, Lyon; 1987. 685. 130 .

6. Jain SP. Etiology of cervix cancer. Directory of Ongoing Research on Cancer Epidemiology. WHO, IRAC, Lyon; 1987; 685: 131.

7. Miller AB. Report on workshop of UICC project on evaluation of screening for cancer. Int J Cancer 1990; 46: 761-70.

8. Aras, RY. Women's education - A cornerstone to attain health for all. Swasth Hind 1984; 1: 23.

9. Miller AB. Cervical Cancer Screening Programmes, Managerial Guidelines. Directory of Ongoing research on Cancer Epidemiology. WHO, IRAC, Lyon; 1987; 685: 168.

10. Sood AK, Sorosky JI. Invasive cervical cancer complicating pregnancy. Obstet Gynecol Clin North Am 1998; 25: 343.

11. Hacker NF, Berek JS, Lagasse LD. Carcinoma of the cervix associated with pregnancy. Obstet Gynecol 1982; 59: 735.

12. Donegan WL. Cancer and pregnancy. CA J Clin 1983; 33: 194.

13. Mikuta JJ. Invasive carcinoma of the cervix in pregnancy. South Med J 1967; 60: 843.

14. Dudan RC, Yon JL, Ford JH. Carcinoma of the cervix and pregnancy. Gynecol Oncol 1973; 1: 283. 